\title{
A review of optimisation models for pedestrian evacuation and design problems
}

\author{
Hendrik Vermuyten ${ }^{\mathrm{a}, *}$, Jeroen Beliën ${ }^{\mathrm{a}, \mathrm{b}}$, Liesje De Boeck ${ }^{\mathrm{a}, \mathrm{b}}$, Genserik \\ Reniers $^{\mathrm{c}, \mathrm{d}}$, Tony Wauters ${ }^{\mathrm{e}}$ \\ ${ }^{a}$ KU Leuven Campus Brussels, Faculty of Economics and Business, Department of \\ Information Management, Modeling and Simulation, Research Centre for Quantitative \\ Business Processes, Warmoesberg 26, B-1000 Brussels, Belgium \\ ${ }^{b}$ KU Leuven, Faculty of Business and Economics, Department of Decision Sciences and \\ Information Management, Research Center for Operations Management, Naamsestraat 69, \\ B-3000 Leuven, Belgium \\ ${ }^{c} K U$ Leuven Campus Brussels, Research Centre for Economics and Corporate \\ Sustainability, Warmoesberg 26, B-1000 Brussel, Belgium \\ ${ }^{d}$ KU Leuven, Research Centre of Energy, Transport and Environment, Naamsestraat 69, \\ B-3000 Leuven, Belgium \\ ${ }^{e} K U$ Leuven, Technology Campus Ghent, Department of Computer Science, CODeS \\ Research Group, Gebroeders De Smetstraat 1, B-9000 Gent, Belgium
}

\begin{abstract}
This article presents a review of the use of optimisation models for pedestrian evacuation and design problems. The articles are classified according to the problem type that is studied, the level of model realism, and the modelling or solution technique. To substantiate the classification criteria and to provide a background for the reader, relevant empirical research and descriptive models (e.g., social-force and cellular automata models) are discussed. We conclude that most of the recent models explicitly include pedestrian dynamics, specifically congestion, but more attention should be given to calibration and implementation of the proposed models. Furthermore, optimisation models could benefit from including some of the modelling techniques used in descriptive models.
\end{abstract}

Keywords:

Pedestrian and crowd behaviour, Optimisation models, Literature review

\footnotetext{
* Corresponding author

Email addresses: hendrik.vermuyten@kuleuven.be (Hendrik Vermuyten), jeroen.belien@kuleuven.be (Jeroen Beliën), liesje.deboeck@econ.kuleuven.be (Liesje De Boeck), genserik.reniers@kuleuven.be (Genserik Reniers), tony.wauters@cs.kuleuven.be (Tony Wauters)
}

Preprint submitted to Safety Science

April 11, 2016 


\section{Introduction}

There are many situations in which a large number of people gathers in a single location. Examples include spectators at music and sports events, commuters in railway and metro stations, and employees in large office buildings.

${ }_{5}$ To ensure the safety and comfort of the people present, a careful design of pedestrian facilities and good crowd management are required. Furthermore, in the event of emergencies, such as a fire, a gas leak, or a bomb threat, the efficient evacuation of the facility is of primary importance. The recent terrorist attacks at the Bataclan theatre in Paris, where 89 people died, and the stampede during this year's Hajj pilgrimage in Mecca, where more than 2,070 people died, illustrate the need for developing good crowd management and emergency evacuation procedures.

The study of pedestrian and evacuation dynamics is very complex, due to the large number of people involved and the non-linear interactions between them, psychological factors influencing human behaviour, and the influence of external factors such as the layout of a pedestrian facility. As a consequence, the topic has received attention from researchers in different fields, including psychologists, sociologists, physicists, computer scientists, and traffic scientists [1].

Three distinct, yet interrelated, research streams can be distinguished. The first stream focuses on the empirical study of pedestrian behaviour and crowd dynamics, while the second is concerned with the development of mathematical models to describe the movement and interactions of pedestrians as realistically as possible [2]. Finally, the third stream of research uses an optimisation-based methodology to develop models which determine optimal evacuation plans or design solutions [3]. Most of the research falls under the first two categories. Several review articles discuss the empirical research on and modelling of pedestrian and evacuation dynamics. Schadschneider et al. [4] provide a summary of the empirical studies and theoretical modelling that has been done and give 
two examples of possible applications of this research. Helbing and Johansson [1] give a similar overview, and additionally discuss research into situations of panic and critical crowd conditions. Schadschneider and Seyfried [5] investigate the quantitative data on pedestrian dynamics for the calibration of evacuation models. They focus on the fundamental diagram (see Section 3.1) and consider 35 the implications for cellular automata models (see Section 4.1). Papadimitriou et al. [6] assess two different topics of research, namely route choice models and crossing behaviour models, which study how pedestrians cross the street under different traffic conditions. Gwynne et al. [7] classify 22 evacuation models based on the nature of the model application, the enclosure representation, the population perspective, and the behavioural perspective. Zheng et al. [8] distinguish seven methodological approaches: cellular automata, lattice-gas, social-force, fluid dynamics, agent-based, game-theoretic models, and experiments with animals. (We give an overview of these approaches in Section 4.1.) They also look at the possibility of modelling heterogeneous individuals, the scale of representation, whether time and space are discrete or continuous, whether a normal or an emergency situation is assumed, and the typical phenomena that the model can represent. In addition, Duives et al. [9] identify eight motion base cases and six self-organising crowd phenomena which a simulation model should be able to reproduce. Furthermore, they look at ten other model characteristics, such as the ability to simulate pressure in crowds and the computational requirements of the model, in order to assess the models' applicability. Their classification distinguishes between cellular automata, social-force, activity-choice, velocity-based, continuum, hybrid, behavioural, and network models. Kalakou and Moura [10] present a general overview of models from different research areas to analyse the design of pedestrian facilities, while Lee et al. [11] focus on models for the evacuation of ships. Finally, Bellomo et al. [12] focus on the mathematical properties of models for pedestrian behaviour. The third category of research has received less attention in the literature. Moreover, to the best of our knowledge, the work of Hamacher and Tjandra [13] is the only review that focuses on optimisation models for evacuation problems. However, most of the models 
they discuss are network models with constant (i.e. density-independent) travel times. This article tries to fill the gap by critically reviewing the different properties of the optimisation models that are currently available for evacuation and design problems and identifying opportunities for future research.

We first searched for literature reviews and articles that discuss general topics related to pedestrian dynamics or evacuation and design problems $[12,9,7,13$, $14,1,10,11,6,4,5,15,16,8]$ and checked the references therein. Next, we used the Web of Knowledge database to find relevant articles. We used combinations of the keywords 'optimisation', 'problem', 'evacuation, 'pedestrian', 'crowd', 'model', 'movement', and 'flow'. No a priori cut-off date was used, since no previous review articles exist that follow our perspective, apart from the work of Hamacher and Tjandra [13]. Articles on the traffic assignment problem and articles on evacuation and design problems which do not focus on pedestrian traffic and crowd dynamics, are not included. This resulted in a broad, but not exhaustive, overview of the current literature on optimisation models for crowd and evacuation dynamics.

In our review, we distinguish between optimisation and non-optimisation articles. The optimisation category consists of all papers that use a methodology to obtain an optimal or a good solution to a specific problem involving crowd so dynamics, such as the efficient evacuation of a building. All articles that describe empirical results or descriptive models for the movement of pedestrians that do not use an optimisation methodology, belong to the non-optimisation category. We only take the optimisation articles into account in our classification process. However, we summarise the empirical research and descriptive modelling approaches in our text in order to give the reader the necessary background information for the discussion of the optimisation models. We ended up with 31 optimisation articles that are included in our classification process.

Figure 1a lists the journals in which most of the articles in this paper have 90 been published. Taking the different types of articles (empirical, descriptive, 


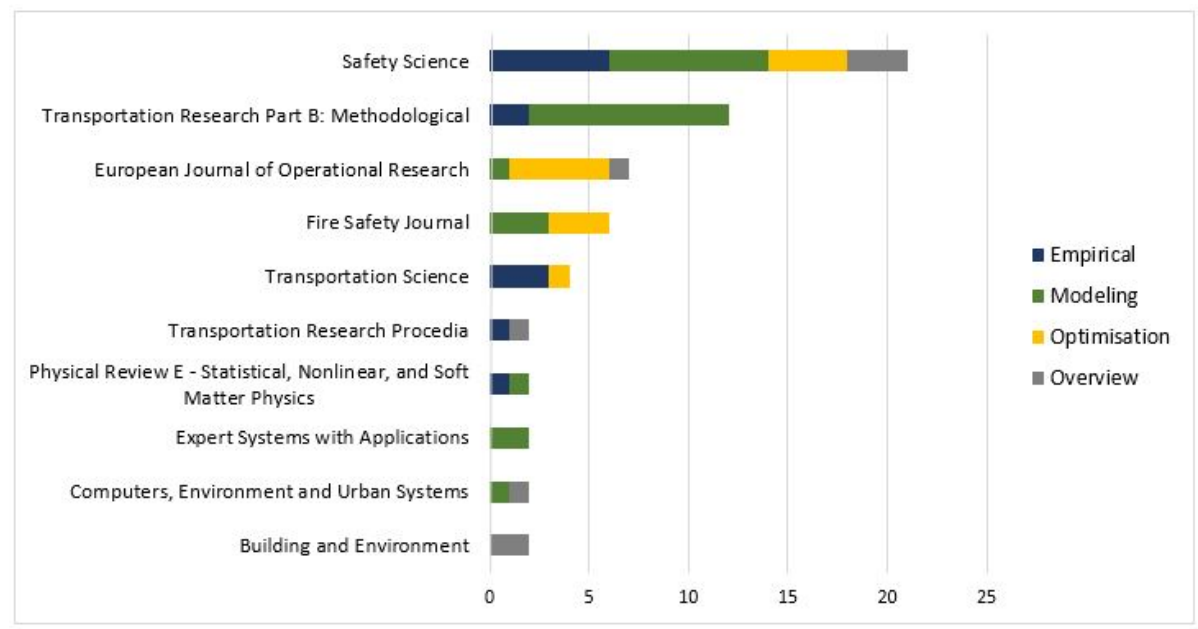

(a) Publications per journal (journals with two or more publications).

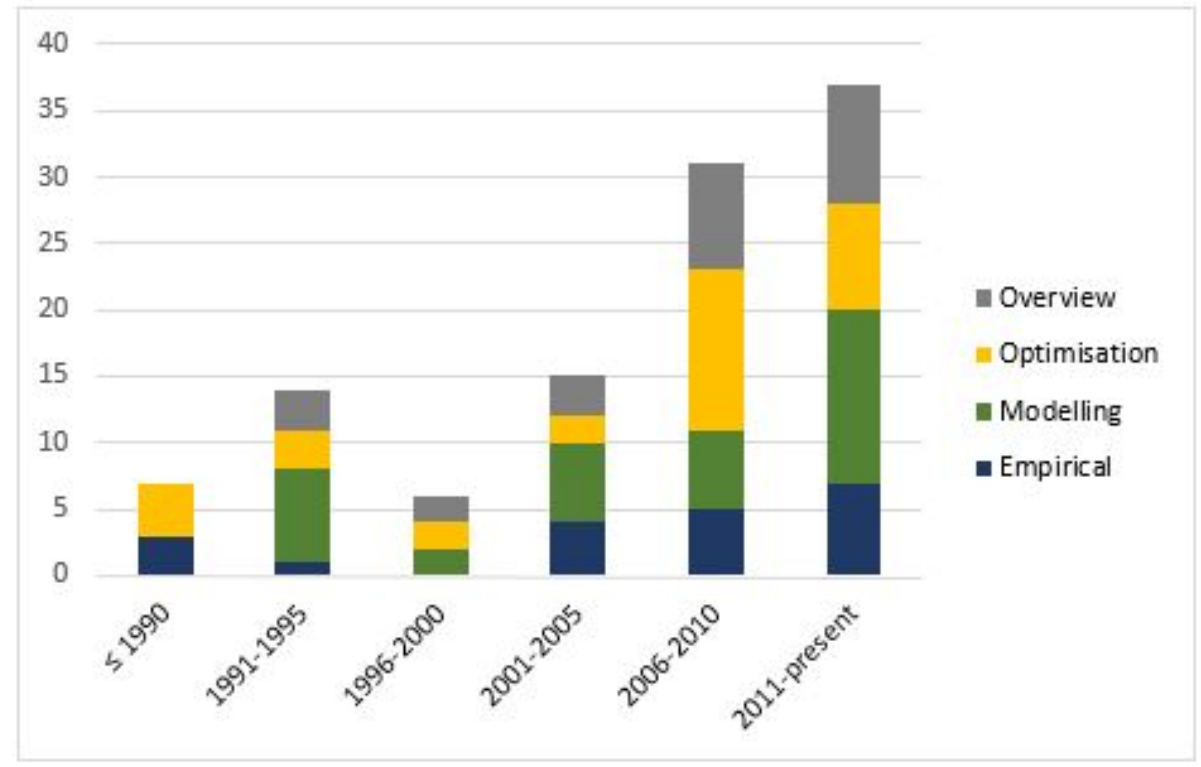

(b) Publications per year.

Figure 1: Overview of publications per journal and per year. 
optimisation, overview) together, Safety Science and Transportation Research Part B: Methodological are the two journals that publish most of the articles related to pedestrian walking behaviour research. Furthermore, Figure 1b gives information on the changing number of articles over the years. It is clear that this research topic has received increasing attention in the last five years.

We use different perspectives for organising the literature. Each section discusses a specific perspective and presents detailed tables in which the relevant articles are categorised. Section 2 discusses the different problem types that are studied in the literature, the criteria used to assess the quality of the resulting solutions, i.e. the objective function measures, and the types of decisions that are considered in the model. The realism of the proposed models and their conformity to empirical results on pedestrian dynamics is investigated in Section 3. Finally, Section 4 analyses the modelling and solution techniques employed to solve the different models. The paper concludes with the main findings and perspectives for future research in Section 5.

\section{Problem type, objective function measures, and decisions consid- ered}

Optimisation models are used to tackle different types of problems related to pedestrian dynamics. As can be seen from Table 1, by far the most attention has been devoted to the development of optimal evacuation plans for pedestrian facilities. Many articles specifically focus on a certain type of pedestrian facility, as this enables researchers to tailor models to the specifics of the environment (e.g. [17]). Most models focus on the evacuation of buildings or large rooms with multiple exits. One of the first articles that studied the building evacuation problem was written by Chalmet, Francis, and Saunders [18] in 1982.

A second type of problem is studied by Johansson and Helbing [19], who look at the problem of finding designs that improve the flow through a bottleneck. Flow is the number of pedestrians who pass through a line segment per meter per second. The study of the influence of design on flow was prompted by 
Table 1: Problem type.

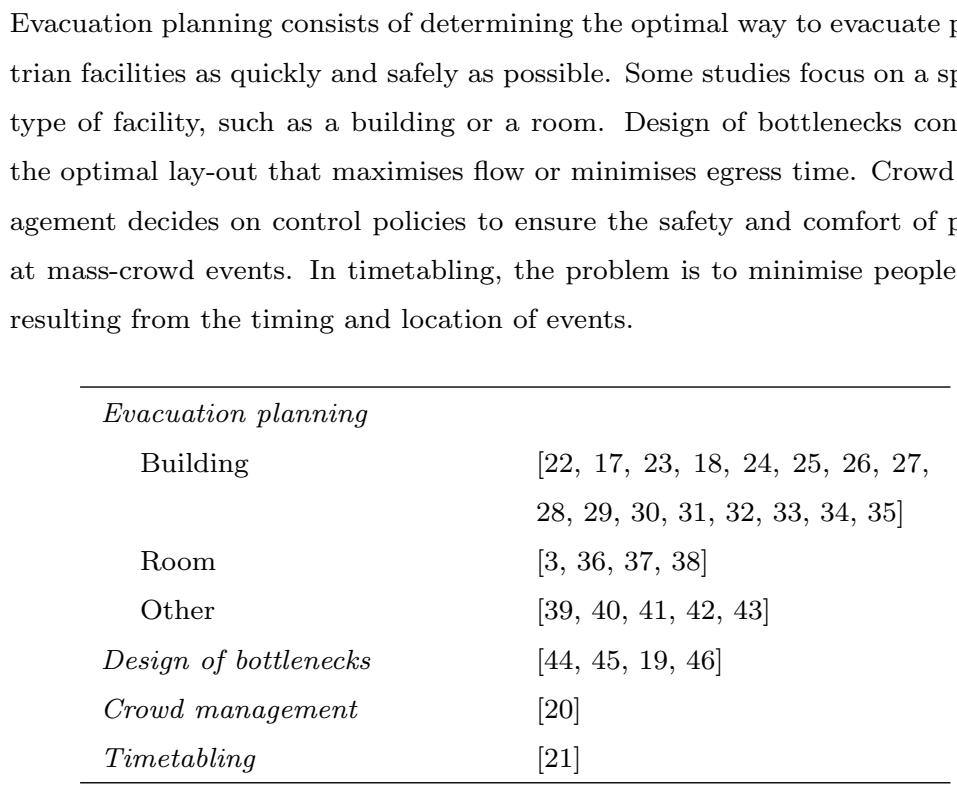

the observation that placing an obstacle in front of the exit can reduce the magnitude of clogging. A genetic algorithm is used to find the configuration that maximises the outflow.

Thirdly, Selim and Al-Rabeh [20] study crowd management to improve the safety and comfort of pedestrians at mass crowd events. Finally, a fourth type of problem is introduced by Vermuyten et al. [21]. They minimise student flows in a university course timetable, since the assignment of lectures to classrooms in the timetable determines student flows and the resulting travel times between consecutive lectures.

In each of these problem types, different objective function measures can be chosen to evaluate the quality of a solution (see Table 2). In the case of evacuation problems, the evacuation time is an important measure of the quality of the proposed plan. Both the average and the maximum evacuation time for all evacuees are used, but the latter is a more popular indicator as it indicates the time that the last person is brought to safety and thus optimises the safety 
Table 2: Objective function measure and problem type.

\begin{tabular}{|c|c|c|c|c|}
\hline & Evacuation & Design & $\begin{array}{l}\text { Crowd man- } \\
\text { agement }\end{array}$ & Timetabling \\
\hline Avg. evac. time & {$[3,18,40]$} & & & \\
\hline Max. evac. time & $\begin{array}{l}{[22,17,23,} \\
18,24,25,26, \\
36,27,28,29, \\
30,31,32,33, \\
39,34,37,35, \\
38,43]\end{array}$ & {$[44,46]$} & & \\
\hline $\begin{array}{l}\text { Number of evac. } \\
\text { people to safety }\end{array}$ & {$[25,29,41]$} & & & \\
\hline Flow & & {$[44,45,19]$} & & \\
\hline Other & {$[29,39,42]$} & & {$[20]$} & {$[21]$} \\
\hline
\end{tabular}

of the least fortunate person. Opasanon and Miller-Hooks [41] also include the number of people evacuated before a certain time. Other researchers minimise the number of people left in the building at each discrete time step [29], minimise the maximum probability of congestion that might occur in the evacuation network [39], or provide the reader with a set of alternatives to choose from [42].

${ }_{140}$ For a further discussion of the many possible performance measures that can be employed for evacuation systems, see Løvås [47]. For design purposes, the maximisation of flow is often used to increase the efficiency of pedestrian facilities, which is important both for normal situations where large pedestrian traffic takes place and for evacuations to reduce congestion and egress times. In the crowd management model [20], the author minimises a penalty function based on the number of people that are denied access at each time interval. Finally, Vermuyten et al. [21] minimise the maximum travel time between consecutive lectures across all different timeslots and series of students in their timetabling problem.

In addition to the objective function measures employed, models can also 
be classified according to the decisions that are included, as is shown in Table 3. The choice of evacuation routes for people to use is the most obvious type of decision included in evacuation models. Some models, however, also incorporate phased evacuation, where different groups of people start evacuation at different times. Phased evacuation is used to reduce congestion on the evacuation routes and consequently improve overall egress times. Zarboutis and Marmaras [42] instead develop generic guidelines for evacuations under different disaster scenarios, instead of proposing a fixed plan for a specific scenario. Furthermore, Talebi and Smith [35] determine the optimal number of nurses to be assigned to each hospital section to achieve the quickest possible evacuation of patients. A different type of decision is modelled by Selim and Al-Rabeh [20], who develop an admission control policy for pedestrians on the Jamarat Bridge to ensure crowd density does not reach hazardous levels. For the category of design problems, Bakuli and Smith [44] determine the optimal widths of exits in a building that maximise throughput, while Berseth et al. [45] derive the optimal placement of obstacles in corridors and at exits to reduce the amount of clogging. Finally, Vermuyten et al. [21] reassign lectures to classrooms in a university course timetable to minimise the maximum travel time of students between consecutive lectures.

\section{Model realism}

It is important that optimisation models represent crowd dynamics in a realistic way and are calibrated with empirical data to provide useful results for evacuation and design purposes. In Subsection 3.1, we first present a summary of the main findings of the empirical research on pedestrian and crowd dynamics. In Subsection 3.2, we discuss the implications of these findings for the development of optimisation models and the problem of parameter calibration. Finally, in Subsections 3.3 and 3.4, we discuss the incorporation of uncertainty into the models and their applicability respectively. 
Table 3: Decisions considered and problem type.

\begin{tabular}{|c|c|c|c|c|}
\hline & Evacuation & Design & $\begin{array}{l}\text { Crowd man- } \\
\text { agement }\end{array}$ & Timetabling \\
\hline Evacuation route choice & $\begin{array}{l}{[22,17,18,} \\
24,25,26,36, \\
27,28,29,30, \\
31,32,33,39, \\
41,34,37,38, \\
43]\end{array}$ & & & \\
\hline Phased evacuation & {$[3,23,40]$} & & & \\
\hline $\begin{array}{l}\text { Generic evacuation } \\
\text { guidelines }\end{array}$ & {$[42]$} & & & \\
\hline $\begin{array}{l}\text { Admission control } \\
\text { policy }\end{array}$ & & & {$[20]$} & \\
\hline $\begin{array}{l}\text { Facility layout \& } \\
\text { location of obstacles }\end{array}$ & & {$[44,45,19,46]$} & & \\
\hline Allocation of staff & {$[35]$} & & & \\
\hline Location of events & & & & {$[21]$} \\
\hline
\end{tabular}

\subsection{Empirical research on pedestrian and crowd dynamics}

A lot of early empirical research focused on the relationship between walking speed, $v\left(\frac{m}{s}\right)$, and density, $\rho\left(\frac{\text { people }}{m^{2}}\right)$, of pedestrian flows. In the same way, the relationship between flow, $q\left(\frac{\text { people }}{m \cdot s}\right)$, and density can be derived, where ${ }_{185} q(\rho)=\rho v(\rho)$. These relationships are called the 'fundamental diagram', because of their importance in determining the optimal dimensions of pedestrian facilities [5]. An early study in 1958 by Hanking and Wright [48] carried out experiments with schoolboys, in which they measured speeds at various concentrations and various passage widths, to obtain the shape of the speed-density and flow-density curves. Then observations were done at a London underground station in order to obtain absolute values for the established relationships. The four parameters that describe this relationship are $\rho_{\max }$, i.e. the maximum density at which walking speed reaches zero, $v_{0}$, i.e. the maximum free walking speed at zero density, and $\rho_{c}$ and $q_{\max }$, which denote the critical density at 

are pushed around by pressure waves in the crowd [1, 50, 51].

Besides the standard fundamental diagram for walking speeds on regular 
Table 4: Parameters for the speed-density and flow-density relationship from various studies.

\begin{tabular}{ccc}
\hline Study & $v_{0}\left(\frac{m}{s}\right)$ & $\rho_{\max }\left(\frac{\text { people }}{m^{2}}\right)$ \\
\hline$[49]$ & 1.30 & 6.60 \\
{$[48]$} & 1.61 & 6.46 \\
{$[51]$} & 0.60 & 10.79 \\
{$[52]$} & 1.40 & 9.00 \\
{$[53]$} & 1.25 & 7.18 \\
{$[54]$} & 1.34 & 5.55 \\
\hline
\end{tabular}

225

horizontal surfaces, walking speeds on stairs have been investigated by some researchers, both descending [62] and ascending [63], as well as for different dimensions (e.g., the height and length of a step) and circumstances (normal and emergency) [64].

A second and related topic of study has been the flow through bottlenecks (e.g., exits). Hoogendoorn and Daamen [65] study the unidirectional flow through a bottleneck for different widths. They observe that pedestrians dynamically form layers inside the bottleneck, where pedestrians are positioned diagonally to the people in front and behind. This phenomenon is called the 'zipper effect', because the layers overlap like interlocking teeth in a zipper. This implies that the capacity of a bottleneck increases in a stepwise manner with the bottleneck width, instead of linearly, depending on how many layers can be formed. Seyfried et al. [66], however, do find a linear relationship between flow and bottleneck width. They argue that the stepwise relationship is based on the faulty assumption that within the bottleneck layers are formed with a constant distance. They also find that jamming occurs below the capacity limit and formulate three hypotheses as an explanation: flow fluctuations, the local organisation of pedestrians, and a preference for larger distances than necessary from the person in front. Helbing et al. [67] and Liu et al. [68] study bidirectional flows through bottlenecks. They find oscillation effects, where multiple pedestrians consecutively pass the bottleneck in a single direction, and clogging effects, where at high densities the movement of pedestrians comes to a halt and 
dangerous pressures are built up in the queues.

Aside from studies that derive quantitative results for pedestrian flows under normal circumstances, other studies have focused on evacuations, since the correct estimation of evacuation times is critical for safety. Olsson and Regan [69] study the evacuation times of three university buildings. They specifically include pre-movement times, i.e. the time people need to realise that they need to evacuate and to decide on a course of action. They argue that the SIMULEX software can be used in evacuation scenario analysis to obtain reliable results. Kady [70] studies the relationship between the density and crawling movement of pedestrians in the event of a fire. The author finds that exit width has a significant impact on crawling speed, while population size is less important. Spearpoint and MacLennan [71] use a Monte Carlo simulation model to investigate the impact of gender, age, and obesity on the evacuation time from a high-rise building.

Furthermore, an important factor of safety concerns the pressures which are experienced by pedestrians in extremely high-density crowds $[1,50]$. Smith and Lim [72] investigate the pressure which people can 'comfortably' endure when pushed against barriers.

Finally, various self-organising crowd phenomena have been observed $[9,1$, 73]. These phenomena are self-organising because they are the result of local interactions between many pedestrians, without any conscious actions of pedestrians to arrive at these phenoma [1]. The most important phenomena are:

Lane formation: In bidirectional flows, pedestrians automatically start forming a number of lanes of varying width, with people in each lane moving in the same direction [4].

Stripe formation for two intersecting flows: When two pedestrian flows intersect, stripes are formed in which pedestrians move forward with the stripes and sidewards within the stripes. This is a result of pedestrians trying to minimize friction with pedestrians moving in opposite directions. For three or more intersecting flows, no stable patterns emerge [67]. 
Stop-and-go waves: At high densities pedestrians cannot move continuously. Instead, the crowd moves in waves [50].

Turbulence: At extremely high densities pedestrians cannot control their own movements anymore, but are pushed around by the forces acting upon them [50].

Herding: When individuals do not have knowledge of the optimal route, they start following others. This happens especially during evacuations [67].

Zipper effect: In a bottleneck individuals move diagonally in front of others such that narrower lanes are formed and the capacity of the bottleneck increases [65].

Faster-is-slower effect: When people keep moving forward when a bottleneck is congested, crowd motion is slowed down by the resulting friction [1].

\subsection{Implications for modelling}

In order to provide realistic results, optimisation models for evacuation or design problems should explicitly incorporate the different empirical results described in the previous section. To asses the realism of the models reviewed, we first focus on three model attributes which capture the different elements of pedestrian and crowd dynamics:

Congestion: Does the model include the relationship between walking speed and density? This means that travel times or flow capacities cannot be assumed to be constants, but should be modelled as endogenous variables dependent on the number of pedestrians present at a certain location.

Bottlenecks: Are bottlenecks such as exits explicitly included in the model? Bottleneck capacities should be based on the width of the bottleneck and the number of people queuing upstream of the bottleneck.

Direction of flow: Does the model distinguish between uni- and bidirectional flows? 
The first part of Table 5 lists the models which explicitly include these that we have reviewed mention calibration of their models. One reason for this is the difficulty of calibrating parameters caused by the significant differences in results that have been obtained in empirical studies [5]. The approach taken 
Table 5: Model realism.

\begin{tabular}{ll}
\hline Incorporation of crowd dynamics & \\
Congestion & {$[3,44,45,22,17,23,25,26,27$,} \\
& $28,19,31,39,34,37,35,46,21$, \\
& $42,38,43]$ \\
Bottlenecks & {$[45,22,23,24,19,31,33,34$,} \\
& $37,35,46,38]$ \\
Direction of flows & {$[45,26,28,46,42,38]$} \\
Reproducing crowd phenomena & {$[22,19,38]$} \\
Calibration & \\
Model tweaking & {$[22,38,43]$} \\
Real-world data & {$[17,23,27,28,37]$} \\
\hline
\end{tabular}

by Venuti and Bruno [60] of including factors that can explain the differences in results in empirical studies of the fundamental diagram, could lead to progress in this area [12].

\subsection{Incorporation of uncertainty into the model}

Evacuations often happen in response to a disaster such as a fire. However, this event usually happens unexpectedly, giving rise to a lot of uncertainty. Indeed, the number of people present at a certain facility and their locations are often not known with certainty. Also, the way the disaster affects the environment, e.g. the propagation of smoke during a fire, and the resulting effects on the evacuation process, can often not be predicted accurately. This has prompted researchers to include uncertainty in their models. We make a distinction between two methods of including uncertainty: predefined probabilities, where parameters or events have a range of possible values or probabilities instead of being deterministic and known, and real-time updating, where the optimisation model uses real-time information on the event to update and adjust the proposed solution. The resulting classification is shown in Table 6 . 
Table 6: Incorporation of uncertainty.

\begin{tabular}{ll}
\hline Predefined probabilities & {$[39,40,41,35,43]$} \\
Real-time updating & {$[24,26,27,28,33,41,34]$} \\
\hline
\end{tabular}

Table 7: Applicability of research.

\begin{tabular}{ll}
\hline No testing & {$[25,29,30]$} \\
Theoretical data & {$[3,44,45,22,17,23,18,24,26$,} \\
& $36,19,32,33,39,40,41,34,37$, \\
& $46,21,42,38,43]$ \\
Real-world data & {$[27,28,31,20,35]$} \\
\hline
\end{tabular}

\subsection{Applicability of the model}

Optimisation models should of course be tested to illustrate their applicabil-

355

\section{Modelling and solution techniques}

In this section, we discuss the different modelling and solution techniques that are proposed in the literature for evacuation problems and design of pedestrian facilities. To provide some background information and ideas for the development of more realistic optimisation models in the future, we first discuss the main techniques used in descriptive models in Subsection 4.1 to realistically represent pedestrian walking behaviour. Afterwards, we compare this with the modelling and solution techniques that are currently used in optimisation models in Subsection 4.2. 


\subsection{Modelling techniques used in descriptive models}

As mentioned above, we briefly discuss some of the approaches that have been developed in the literature for the modelling of pedestrian behaviour and crowd dynamics. We do not intend to give an exhaustive overview of the different modelling techniques or an in-depth discussion of the properties of each model that is included. The interested reader can find detailed assessments of the existing modelling approaches and simulation models in [9], [6], and [8].

\subsubsection{Continuum models}

Continuum models are macroscopic simulation models. Pedestrians are not represented individually; instead crowds are described as a fluid using average quantities such as the density at a given location. Mathematically, these models consist of a system of partial differential equations, expressing the relationship between average speed, flow, and density at a given location and time [12]. Both time and space are continuous. A distinction can be made between firstorder models, which only include an equation for the conservation of mass, and second-order models, which also include a momentum balance equation [12]. Since it is computationally efficient, the continuum approach is often used when very large crowds need to be modelled or when only an estimation of the average quantities is required. One of the first authors that applied these continuum models to pedestrian traffic was Hughes [74]. He develops a first-order model based on three hypotheses: (i) pedestrians' speed is determined by the local density at their location, (ii) pedestrians' movement is perpendicular to lines of constant potential, and (iii) pedestrians want to take the path with the shortest travel time, but only if the density on this path is not too high. Huang et al. [75] prove that Hughes' model satisfies the reactive dynamic user equilibrium, which means that pedestrians choose the route that minimises their instantaneous travel cost to the destination. They also develop an efficient solution method to solve the model. Hoogendoorn and Bovy [76] present a continuum model which applies to different types of traffic, i.e. both vehicular and pedestrian traffic. They develop the concept of generalised phase-space density, to include 

purposes, while others use smaller cells where each pedestrian occupies multiple cells, to allow for a greater degree of detail (e.g. [85]). Time is discretised and 
at each time step, pedestrians either move to a neighbouring cell or remain at

their current location. The decision taken by a pedestrian depends on the status of the adjacent cells and is based on a predefined set of rules. Updating of cells can be executed either sequentially (e.g. [85]) or in parallel (e.g. [84]), in which case movements can only be executed when all conflicts between pedestrians are resolved. One of the first cellular automata models for the simulation of pedestrian movements was developed by Blue and Adler [84]. The authors focus on the various phenomena observed in bidirectional flows. Guo et al. [85] develop two route choice models, for the case of good and bad visibility respectively. Pereira et al. [86] explicitly include the relationship between average speed of a pedestrian and the density in the model. An advantage of the approach is its computational efficiency.

\subsubsection{Agent-based models}

Agent-based models take a bottom-up approach as well, where only the behaviour of individual pedestrians is modelled and the resulting interactions between them determine the macroscopic behaviour. Agent-based models can use both discrete and continuous time and space representations. Each agent can have a unique set of behavioural rules, which allows for modelling heterogeneity in the population (e.g., different preferred walking speeds for old and young people). A disadvantage of this flexibility is the high computational cost of running the model. Antonini et al. [87] use a discrete choice framework in which pedestrians choose a direction and speed based on the utility of each of the alternatives. This utility is influenced by the presence of other pedestrians. Chooramun et al. [88] combine three space representations (continuous space, fine network, and coarse network) into a single model to achieve an optimal trade-off between computational efficiency and model realism. Behaviour of agents is based on a different set of rules at each representation level. The MOBEDIC tool developed by Doheny and Fraser [89] models the actions of people in specific emergency situations, specifically focusing on the evacuation of an offshore environment. EXODUS is a similar software tool, developed by 
Galea and Perez Galparsoro, intended for the evacuation of mass-transport vehicles such as aircraft [90]. It is also able to simulate crawling movement during evacuations [91]. A third software tool, developed for simulating the evacuation of geometrically complex buildings, is the SIMULEX model of Thompson and Marchant [92, 93, 94]. Recently, Wagner and Agrawal [95] developed an agentbased model for the evacuation of concert venues. The propagation of fire and smoke is included in the model and influences the route choice behaviour of individuals. However, there are still many challenges involved in the development of agent-based models, see Crooks et al. [96] for a discussion.

\subsubsection{Social-force models}

A third set of microscopic models consists of the so-called social-force models. In this type of model, pedestrians have a desired velocity in the direction of their destination and their acceleration (deceleration) is the result of different forces. An individual experiences an attractive force in the direction of his target destination, and repulsive forces from obstacles (e.g. walls) and other pedestrians. Time and space are modelled in a continuous way. The social-force model was developed by Helbing $[58,59]$. The model reproduces well-known self-organising crowd phenomena such as lane formation in bidirectional flows and oscillatory effects at bottlenecks. Langston et al. [97] represent pedestrians by three intersecting circles instead of a single circle, to incorporate the rotation of the pedestrians into the model. The model is realistic for dense crowd flow scenarios, but more complex scenarios are not yet fully realistically represented. Yuen and Lee [98] extend the social-force model to include overtaking behaviour, where pedestrians with a higher desired velocity catch up with and move past pedestrians heading in the same direction with a lower desired velocity. Qu et al. [99] also use a three-circle representation to model rotation and extend the social-force model to describe pedestrian movement on stairs. 


\subsubsection{Game-theoretic models}

Hoogendoorn and Bovy [100] use the theory of differential games to describe the walking behaviour of pedestrians. In this model, pedestrians predict the behaviour of other pedestrians based on the current state and anticipated actions of other pedestrians in their neighbourhood (predictive dynamic user equilibrium principle). They base their pedestrian walking behaviour model on a clear theoretical foundation based on the micro-economic notion of subjective utility maximisation. The same authors develop a comprehensive theory of pedestrian activity and path determination in the two-dimensional space [101]. Huang et al. [75] instead use a reactive user equilibrium principle in which pedestrians only evaluate the immediate conditions of their environment without anticipating the behaviour of pedestrians in their surroundings [102]. Their model is an extension of the macroscopic model of Hughes [74]. Lachapelle and Wolfram [103] present a pedestrian crowd model based on the theory of mean field games. The model is macroscopic, i.e. it describes crowd behaviour in terms of aggregates, but it is based on a realistic microscopic model in the sense that it considers smart pedestrians with rational expectations. Pedestrians are represented as agents having preferences (i.e. they want to maximize their utility) and perform strategic interactions within the crowd. They also anticipate the future. This approach is similar to that of Hoogendoorn and Bovy [100], but an advantage of the former model is its lower computational cost as compared to microscopic simulation models.

\subsection{Modelling techniques used in optimisation models}

Table 8 lists the different papers according to the optimisation modelling technique that is used.

Many early models focus on exact methods, such as standard network flow models and dynamic programming (i.e. shortest path) to determine optimal evacuation plans. Chalmet et al. [18] represent a building by a graph in which the nodes denote the rooms and the arcs the connections, i.e. doors, between them. They use a dynamic network flow algorithm to simultaneously minimise 
the average evacuation time, the maximal evacuation time, and to maximise the total number of people evacuated by a given time. Another example is the EVACNET+ software developed by Kisko and Francis [32], which uses a network flow algorithm to determine optimal evacuation routes.

Additionally, many authors develop a dedicated algorithm to solve their respective models. Ding [36] presents an evacuation model where people are assigned to different exit routes, each with a certain length and width, such that the total evacuation time is minimised. The author derives an expression for the number of people that should be assigned to each exit route, based on the observation that the evacuation time over all routes should be equal, since it is the last person's egress time that should be minimised. A similar problem is described by Pursals and Garzón [37]. The expressions proposed by Nelson and McLennan [104] are used to model the movement of people, i.e. to represent the non-linear relationship between density and travel time on a given route. 530 The authors then adapt the algorithm of Brown [105] for the knapsack sharing problem to solve their problem.

However, while computationally efficient, these models do not model pedestrian dynamics well, as they assume capacities and arc-traverse times to be constant instead of density-dependent. In recent years, the development of more 535 realistic models which better represent crowd phenomena, has shifted attention towards the use of queuing models and heuristics on the one hand and the use of simulation on the other hand to cope with the increased complexity.

Queuing models can represent buildings as a graph where nodes correspond to rooms or bottlenecks and arcs correspond to the connections between them $[44,35]$, or by a lattice where each cell can be occupied by a number of people and has a queuing process associated with it [26]. The travel and waiting time are modelled by the queuing process at each node. The service rate is a function of the number of people present because of the inverse relationship between walking speed and density of pedestrians. The advantage of these models is that they include this non-linear relationship, instead of assuming constant travel times and capacities, while at the same time being computationally efficient to solve. 
Deng et al. [26] combine Markov Decision Process models and queuing theory to model the evacuation of a building. The Markov process describes the typical egress behaviour of an agent, while a queue at each building node is used to

run a new population is created from the previous one, until a stopping criterion is reached. The solution with the lowest evacuation time then represents the 
Table 8: Solution technique.

\begin{tabular}{ll}
\hline Mathematical programming & \\
Dynamic programming - Shortest path & {$[24,27,34]$} \\
Network flow transshipment algorithm & {$[22,18,25,32]$} \\
Integer programming & {$[31,39,21]$} \\
Chance constraint programming & {$[40]$} \\
Heuristic & \\
Simulated annealing & {$[17,23]$} \\
Genetic algorithm & {$[3,19]$} \\
Simulation & {$[3,28,38]$} \\
Cellular automata & {$[46,42]$} \\
Agent-based modelling & {$[45,26,19,43]$} \\
Other & {$[44,26,35]$} \\
Queuing & {$[25,36,28,29,30,31,33,41,37,20]$} \\
Dedicated algorithm &
\end{tabular}

best evacuation plan that has been found.

Similar techniques are used by Johansson and Helbing [19] and Tavares [46] for design problems. Johansson and Helbing [19] use a genetic algorithm in combination with the social-force model to find an improved layout to increase the flow through a bottleneck.

Most articles that we studied do not discuss the computational requirements of their models. An exception is the models by Georgoudas et al. [28] and Li and $\mathrm{Xu}$ [33], which track pedestrians in real-time and suggest rerouting pedestrians based on anticipated congestion at exits. In this case, computational efficiency is of the utmost importance in order to be able to determine optimised evacuation routes in real-time.

\section{Discussion and conclusion}

In this article, we have reviewed optimisation models from the field of pedestrian walking behaviour and crowd dynamics. These models are used for a wide 
range of evacuation and design problems. We have also discussed the relevant empirical research and descriptive modelling techniques to provide a background for the reader and to substantiate the criteria that are used in the assessment of the different models.

Currently, most of the attention is directed to the development of optimal evacuation plans, followed by the effective design of pedestrian facilities. However, there are other interesting problems related to pedestrian flows which have not yet received much attention in the literature, such as crowd management under normal conditions. An example is the minimisation of flows resulting from the timing and location of certain events, such as the assignment of lectures to rooms and timeslots in a university timetable, the scheduling of acts at music festivals, or the planning of different disciplines at large sports events, to ensure the safety (i.e., crowd densities do not reach hazardous levels) and comfort (i.e., people do not have to walk large distances or through high-density crowds and can reach their destinations in time) of the people present.

While many of the earlier models concerning evacuation problems did not include the fundamental relationship between walking speed and crowd density, and instead assumed constant travel times, most of the recent articles represent these dynamics in their models. By way of contrast, the calibration of models should receive more attention in future work. However, calibration is still difficult because of the lack of consensus between data of different empirical studies. More research is needed in this area to reconcile or explain the contradictory 615 results obtained in experiments.

Closely related to this is the validation and application of optimisation models. Currently, most authors only test their models on theoretical data. To implement the models in practice, it is important that their results and predictions closely resemble real-world values. Furthermore, practitioners could benefit if authors describe the different challenges and pitfalls in implementing their models.

Finally, there currently is a discrepancy between the techniques used in descriptive models and those used in optimisation models. The former are mostly 
variants of microscopic simulation models, because they seek to represent pedes-

trian dynamics as realistically as possible. By way of contrast, the latter gravitate towards network models in combination with flow transshipment algorithms or queuing processes, because of their mathematical tractability. Some of the recent models use an iterative process where a heuristic searches for good solutions, which are consequently tested by a simulation model that represents the resulting crowd dynamics in a realistic way. Future research should focus on integrating techniques of descriptive models within an optimisation framework to find the optimal trade-off between model realism and tractability.

\section{Acknowledgement}

This research was sponsored by the Research Foundation - Flanders (FWO) contract no. G072316N.

\section{References}

[1] D. Helbing, A. Johansson, Pedestrian, Crowd and Evacuation Dynamics, in: Encyclopedia of complexity and System Science, Vol. 16, 2010, pp. 6476-6495. arXiv:arXiv:1309.1609v1, doi:10.1007/978-3-642-04504-2.

URL http://www. springerlink.com/index/10.1007/ $978-3-642-04504-2$

[2] K. Teknomo, Microscopic Pedestrian Flow Characteristics: Development of an Image Processing Data Collection and Simulation Model, Ph.D. thesis (2002).

[3] A. Abdelghany, K. Abdelghany, H. Mahmassani, W. Alhalabi, Modeling framework for optimal evacuation of large-scale crowded pedestrian facilities, European Journal of Operational Research 237 (3) (2014) 1105-1118. doi:10.1016/j.ejor.2014.02.054.

URL http://dx.doi.org/10.1016/j.ejor.2014.02.054 
[4] A. Schadschneider, W. Klingsch, H. Kluepfel, T. Kretz, C. Rogsch, A. Seyfried, Evacuation Dynamics: Empirical Results, Modeling and Applications, in: Encyclopedia of Complexity and System Science, 2008, pp. 517-550. arXiv:0802.1620, doi:10.1007/978-0-387-30440-3\{\_\}187.

[5] A. Schadschneider, A. Seyfried, Empirical results for pedestrian dynamics and their implications for cellular automata models, in: Pedestrian Behavior - Models, Data Collection and Applications, 2009, pp. 27-44. arXiv: 1007.4058.

URL http://arxiv.org/abs/1007.4058

[6] E. Papadimitriou, G. Yannis, J. Golias, A critical assessment of pedestrian behaviour models, Transportation Research Part F: Traffic Psychology and Behaviour 12 (3) (2009) 242-255. doi:10.1016/j.trf .2008.12.004. URL http://dx.doi.org/10.1016/j.trf.2008.12.004

[7] S. Gwynne, E. Galea, M. Owen, P. Lawrence, L. Filippidis, A review of the methodologies used in the computer simulation of evacuation from the built environment, Building and Environment 34 (6) (1999) 741-749. doi:10.1016/S0360-1323(98)00057-2.

[8] X. Zheng, T. Zhong, M. Liu, Modeling crowd evacuation of a building based on seven methodological approaches, Building and Environment 44 (3) (2009) 437-445. doi:10.1016/j.buildenv.2008.04.002.

[9] D. C. Duives, W. Daamen, S. P. Hoogendoorn, State-of-the-art crowd motion simulation models, Transportation Research Part C: Emerging Technologies 37 (1) (2013) 193-209. doi:10.1016/j.trc.2013.02.005. URL http://dx.doi.org/10.1016/j.trc.2013.02.005

[10] S. Kalakou, F. Moura, Bridging the Gap in Planning Indoor Pedestrian Facilities, Transport Reviews: A Transnational Transdisciplinary Journal 34 (4) (2014) 474-500. doi:10.1080/01441647.2014.915441. 
URL http://www.tandfonline.com/doi/abs/10.1080/01441647.

[11] D. Lee, H. Kim, J.-H. Park, B.-J. Park, The current status and future issues in human evacuation from ships, Safety Science 41 (10) (2003) 861876. doi:10.1016/S0925-7535(02)00046-2.

[12] N. Bellomo, B. Piccoli, A. Tosin, Modeling Crowd Dynamics From a ComSciences 22 (2) (2012) 1230004. doi:10.1142/S0218202512300049.

[13] H. W. Hamacher, S. a. Tjandra, Mathematical modelling of evacuation problems: a state of the art., in: Pedestrian and Evacuation Dynamics, 2002, pp. 227-266. doi:citeulike-article-id:6650160.

[14] G.-q. He, Y. Yang, Z.-h. Chen, C.-h. Gu, Z.-g. Pan, A review of behavior mechanisms and crowd evacuation animation in emergency exercises, Journal of Zhejiang University - SCIENCE C (Computers \& Electronics) 14 (7) (2013) 477-485. doi:10.1631/jzus. CIDE1301. URL http://link.springer.com/10.1631/jzus.CIDE1301

[15] J. D. Sime, Crowd psychology and engineering, Safety Science 21 (1) (1995) 1-14. doi:10.1016/0925-7535(96)81011-3.

[16] R. J. C. Stanton, G. K. Wanless, Pedestrian movement, Safety Science 18 (4) (1995) 291-300. doi:10.1016/0925-7535(94)00037-4.

[17] E. M. Cepolina, A methodology for defining building evacuation routes, Civil Engineering and Environmental Systems 22 (1) (2005) 29-47. doi: $10.1080 / 10286600500049946$.

[18] L. G. Chalmet, R. L. Francis, P. B. Saunders, Network Models for Building Evacuation, Management Science 28 (1) (1982) 86-105.

[19] A. Johansson, D. Helbing, Pedestrian flow optimization with a genetic algorithm based on Boolean grids, in: Pedestrian and evacuation dynamics, 2005, pp. 267-272. 
[20] S. Z. Selim, a. H. Al-Rabeh, On the Modeling of Pedestrian Flow on the Jamarat Bridge, Transportation Science 25 (4) (1991) 257-263. doi: $10.1287 / \operatorname{trsc} .25 .4 .257$.

[21] H. Vermuyten, S. Lemmens, J. Beliën, I. Marques, Developing compact course timetables with optimized student flows, European Journal of Operational Research, (in press).

[22] A. Borrmann, A. Kneidl, G. Köster, S. Ruzika, M. Thiemann, Bidirectional coupling of macroscopic and microscopic pedestrian evacuation models, Safety Science 50 (8) (2012) 1695-1703. doi:10.1016/j.ssci. 2011.12 .021$.

[23] E. M. Cepolina, Phased evacuation: An optimisation model which takes into account the capacity drop phenomenon in pedestrian flows, Fire Safety Journal 44 (4) (2006) 532-544. doi:10.1016/j.firesaf. 2008. 11.002 .

[24] P. H. Chen, F. Feng, A fast flow control algorithm for real-time emergency evacuation in large indoor areas, Fire Safety Journal 44 (5) (2009) 732 740. doi:10.1016/j.firesaf.2009.02.005.

[25] W. Choi, H. Hamacher, S. Tufekci, Modeling of building evacuation problems by network flows with side constraints, European Journal of Operational Research 35 (1) (1988) 98-110. doi: 10.1016/0377-2217(88)90382-7.

URL http://www.sciencedirect.com/science/article/pii/ 0377221788903827

[26] K. Deng, W. Chen, P. G. Mehta, S. P. Meyn, Resource pooling for optimal evacuation of a large building, Proceedings of the IEEE Conference on Decision and Control 47 (2008) 5565-5570. doi:10.1109/CDC. 2008. 4739428. 
[27] R. F. Fahy, EXIT 89 - An Evacuation Model for High Rise Buildings

- Model Description and Example Applications (1994). doi:10.3801/ IAFSS.FSS.4-657.

[28] I. G. Georgoudas, G. C. Sirakoulis, I. T. Andreadis, An Anticipative Crowd Management System Preventing Clogging in Exits During Pedestrian Evacuation Processes, IEEE Systems Journal 5 (1) (2010) 129-141. doi:10.1109/JSYST . 2010.2090400.

[29] B. Hoppe, E. Tardos, Polynomial time algorithms for some evacuation problems, in: Proceedings of the Fifth Annual SIAM Symposium on Discrete Algorithms, 1994, pp. 433-441.

[30] B. Hoppe, E. Tardos, The Quickest Transshipment Problem, Mathematics of Operations Research 25 (1) (2000) 36-62. doi:10.1287/moor.25.1. 36.15211.

[31] J. Kang, I.-J. Jeong, J.-B. Kwun, Optimal facility-final exit assignment algorithm for building complex evacuation, Computers \& Industrial Engineering 85 (2015) 169-176. doi:10.1016/j.cie.2015.03.012.

URL http://www.sciencedirect.com/science/article/pii/ S0360835215001217

[32] T. M. Kisko, R. L. Francis, EVACNET+: A computer program to determine optimal building evacuation plans, Fire Safety Journal 9 (2) (1985) 211-220. doi:10.1016/0379-7112(85)90009-8.

[33] N. Li, Y. Xu, Evacuation Modeling From the Control Perspective and Corresponding Sequential-Based Optimal Evacuation Guidance, IEEE Transactions on Control Systems Technology 22 (3) (2014) 1094-1102. doi:10.1109/TCST.2013.2272558.

URL http://www. scopus.com/inward/record.url?eid=2-s2. 0-84899944107\{\&\} partnerID=tZOtx3y1 
[34] I. Park, G. U. Jang, S. Park, J. Lee, Time-dependent optimal routing in micro-scale emergency situation, Proceedings - IEEE International Conference on Mobile Data Management 10 (2009) 714-719. doi: 10.1109/MDM. 2009.122.

[35] K. Talebi, J. Smith, Stochastic Network Evacuation Models, Computers \& Operations Research 12 (6) (1985) 559-577.

[36] A. W. Ding, Implementing real-time grouping for fast egress in emergency, Safety Science 49 (10) (2011) 1404-1411. doi:10.1016/j.ssci.2011.06. 006.

URL http://dx.doi.org/10.1016/j.ssci.2011.06.006

[37] S. C. Pursals, F. G. Garzón, Optimal building evacuation time considering evacuation routes, European Journal of Operational Research 192 (2) (2009) 692-699. doi:10.1016/j.ejor.2007.10.004.

[38] H. Zhao, Z. Gao, Reserve capacity and exit choosing in pedestrian evacuation dynamics, Journal of Physics A: Mathematical and Theoretical 43 (10) (2010) 105001. doi:10.1088/1751-8113/43/10/105001.

[39] G. J. Lim, M. Rungta, M. R. Baharnemati, Reliability analysis of evacuation routes under capacity uncertainty of road links, IIE Transactions 47 (1) (2015) 50-63. doi:10.1080/0740817X.2014.905736.

[40] M. Ng, S. T. Waller, Reliable evacuation planning via demand inflation and supply deflation, Transportation Research Part E: Logistics and Transportation Review 46 (6) (2010) 1086-1094. doi:10.1016/j.tre. 2010.04 .001$.

URL http://dx.doi.org/10.1016/j.tre.2010.04.001

[41] S. Opasanon, E. Miller-Hooks, The Safest Escape problem, Journal of the Operational Research Society 60 (12) (2009) 1749-1758. doi:10.1057/ jors.2008.122. 
[42] N. Zarboutis, N. Marmaras, Design of formative evacuation plans using agent-based simulation, Safety Science 45 (9) (2007) 920-940. doi:10. 1016/j.ssci.2006.08.029.

[43] X. Zheng, M. Liu, Forecasting model for pedestrian distribution under emergency evacuation, Reliability Engineering \& System Safety 95 (11) (2010) 1186-1192. doi:10.1016/j.ress.2010.07.005.

URL http://dx.doi.org/10.1016/j.ress.2010.07.005

[44] D. L. Bakuli, J. M. Smith, Resource allocation in state-dependent emergency evacuation networks, European Journal of Operational Research 89 (3) (1996) 543-555.

[45] G. Berseth, M. Usman, B. Haworth, M. Kapadia, P. Faloutsos, Environment optimization for crowd evacuation, Computer Animation and Virtual Worlds 26 (3-4) (2015) 377-386. doi:10.1002/cav.

URL http://www.ingentaconnect.com/content/jws/cav/2009/ 00000020/F0020002/art00003

[46] R. M. Tavares, Design for horizontal escape in buildings: The use of the relative distance between exits as an alternative approach to the maximum travel distance, Safety Science 48 (10) (2010) 1242-1247. doi:10.1016/ j.ssci.2010.03.009.

URL http://dx.doi.org/10.1016/j.ssci.2010.03.009

[47] G. G. Løvås, On performance measures for evacuation systems, European Journal of Operational Research 85 (2) (1995) 352-367. doi:10.1016/ 0377-2217 (94)00054-G.

[48] B. Hankin, R. Wright, Passenger Flow in Subways, OR 9 (2) (1958) 81-88. doi:10.1093/brain/awt057.

[49] J. Fruin, Pedestrian Planning and Design, Metropolitan Association of Urban Designers and Environmental Planners, New York, 1971. 
[50] D. Helbing, A. Johansson, H. Z. Al-Abideen, Dynamics of crowd disasters: An empirical study, Physical Review E - Statistical, Nonlinear, and Soft Matter Physics 75 (4) (2007) 1-7. arXiv:0701203, doi: 10.1103/PhysRevE. 75.046109.

[51] A. Johansson, D. Helbing, H. Z. Al-abideen, S. Al-bosta, From Crowd Dynamics to Crowd Safety: A Video-Based Analysis, Advances in Complex Systems 11 (2008) 479-527. arXiv:arXiv:0810.4590v1.

[52] M. Mōri, H. Tsukaguchi, A new method for evaluation of level of service in pedestrian facilities, Transportation Research Part A: General 21 (3) (1987) 223-234. doi:10.1016/0191-2607(87)90016-1.

[53] A. Polus, L. Joseph, A. Ushpiz, Pedestrian flow and level of service, Journal of Transportation Engineering 109 (1983) 46-56.

[54] A. Seyfried, B. Steffen, W. Klingsch, M. Boltes, The Fundamental Diagram of Pedestrian Movement Revisited, Journal of Statistical Mechanics P10002 (2005) 13. arXiv:0506170, doi:10.1088/1742-5468/2005/10/ P10002.

URL http://arxiv.org/abs/physics/0506170

[55] V. Predtechenskii, A. Milinskii, Planning for foot traffic flow in buildings, Amerind Publishing, New Dehli, 1978.

[56] D. Oeding, Verkehrsbelastung und Dimensionierung von Gehwegen und anderen Anlagen des Fugängerverkehrs, Forschungsbericht 22, Technische Hochschule Braunschweig (1963).

[57] G. Flötteröd, G. Lämmel, Bidirectional pedestrian fundamental diagram, Transportation Research Part B: Methodological 71 (2015) 194-212. doi:10.1016/j.trb.2014.11.001.

URL http://linkinghub.elsevier.com/retrieve/pii/ S0191261514001908 
[58] D. Helbing, A mathematical model for the behavior of pedestrians, Behavioral Science 36 (4) (1991) 298-310.

[59] D. Helbing, P. Molnar, Social force model for pedestrian dynamics, Physical Review E 51 (5) (1995) 4282-4286.

[60] F. Venuti, L. Bruno, An interpretative model of the pedestrian fundamental relation, Comptes Rendus - Mecanique 335 (4) (2007) 194-200. doi:10.1016/j.crme.2007.03.008.

[61] R. Galiza, L. Ferreira, A methodology for determining equivalent factors in heterogeneous pedestrian flows, Computers, Environment and Urban Systems 39 (2013) 162-171. doi:10.1016/j.compenvurbsys.2012.08. 003.

URL http://dx.doi.org/10.1016/j.compenvurbsys.2012.08.003

[62] J. Ma, W. G. Song, W. Tian, S. M. Lo, G. X. Liao, Experimental study on an ultra high-rise building evacuation in China, Safety Science 50 (8) (2012) 1665-1674. doi:10.1016/j.ssci.2011.12.018.

[63] J. H. Lam, J. K. Yuen, E. W. Lee, R. Y. Lee, Experimental study on upward movement in a high-rise building, Safety Science 70 (2014) 397-405. doi:10.1016/j.ssci.2014.07.011.

[64] L. Yang, P. Rao, K. Zhu, S. Liu, X. Zhan, Observation study of pedestrian flow on staircases with different dimensions under normal and emergency conditions, Safety Science 50 (5) (2012) 1173-1179. doi: 10.1016/j.ssci.2011.12.026.

URL http://dx.doi.org/10.1016/j.ssci.2011.12.026

[65] S. P. Hoogendoorn, W. Daamen, Pedestrian Behavior at Bottlenecks, Transportation Science 39 (2) (2005) 147-159. doi:10.1287/trsc. 1040. 0102. 
[66] A. Seyfried, T. Rupprecht, O. Passon, B. Steffen, W. Klingsch, M. Boltes, New insights into pedestrian flow through bottlenecks, Transportation Science 43 (3) (2009) 395-406. arXiv:0702004, doi:10.1287/trsc.1090. 0263.

URL http://arxiv.org/abs/physics/0702004

${ }_{875}^{6}$ [67] D. Helbing, L. Buzna, A. Johansson, T. Werner, Self-organized pedestrian crowd dynamics: Experiments, simulations, and design solutions, Transportation Science 39 (1) (2005) 1-24. doi:10.1287/trsc.1040.0108.

URL http://discovery.ucl.ac.uk/150154/

[68] X.-d. Liu, W.-g. Song, W. Lv, Empirical Data for Pedestrian Counterflow through Bottlenecks in the Channel, Transportation Research Procedia 2 (2014) 34-42. doi:10.1016/j.trpro.2014.09.006.

URL http://www.sciencedirect.com/science/article/pii/ S2352146514000428

[69] P. A. Olsson, M. a. Regan, A comparison between actual and predicted evacuation times, Safety Science 38 (2) (2001) 139-145. doi:10.1016/ S0925-7535(00) 00064-3.

[70] R. a. Kady, The development of a movement-density relationship for people going on four in evacuation, Safety Science 50 (2) (2012) 253-258. doi:10.1016/j.ssci.2011.08.058. URL http://dx.doi.org/10.1016/j.ssci.2011.08.058

[71] M. Spearpoint, H. a. MacLennan, The effect of an ageing and less fit population on the ability of people to egress buildings, Safety Science 50 (8) (2012) 1675-1684. doi:10.1016/j.ssci.2011.12.019.

URL http://dx.doi.org/10.1016/j.ssci.2011.12.019

${ }_{895}$ [72] R. a. Smith, L. B. Lim, Experiments to investigate the level of 'comfortable' loads for people against crush barriers, Safety Science 18 (4) (1995) 329-335. doi : 10.1016/0925-7535 (94)00052-5. 
[73] M. Moussaïd, D. Helbing, S. Garnier, A. Johansson, M. Combe, G. Theraulaz, Experimental study of the behavioural mechanisms underlying selforganization in human crowds., in: Proceedings of The Royal Society B, Vol. 276, 2009, pp. 2755-2762. arXiv:0908.3131, doi:10.1098/rspb. 2009.0405.

[74] R. L. Hughes, A continuum theory for the flow of pedestrians, Transportation Research Part B: Methodological 36 (6) (2002) 507-535. doi : 10.1016/S0191-2615(01)00015-7.

[75] L. Huang, S. Wong, M. Zhang, C.-W. Shu, W. H. Lam, Revisiting Hughes dynamic continuum model for pedestrian flow and the development of an efficient solution algorithm, Transportation Research Part B: Methodological 43 (1) (2009) 127-141. doi:10.1016/j.trb.2008.06.003. URL http://dx.doi.org/10.1016/j.trb.2008.06.003

[76] S. P. Hoogendoorn, P. H. L. Bovy, Generic gas-kinetic traffic systems modeling with applications to vehicular traffic flow, Transportation Research Part B: Methodological 35 (4) (2001) 317-336. doi:10.1016/ S0191-2615(99) 00053-3.

[77] C. Appert-Rolland, P. Degond, S. Motsch, Two-way multi-lane traffic model for pedestrians in corridors, Networks and Heterogeneous Media 6 (2011) 351-381. arXiv:1105.0203, doi:10.3934/nhm.2011.6.351. URL http://arxiv.org/abs/1105.0203

[78] F. S. Hänseler, M. Bierlaire, B. Farooq, T. Mühlematter, A macroscopic loading model for time-varying pedestrian flows in public walking areas, Transportation Research Part B: Methodological 69 (2014) 60-80. doi:10.1016/j.trb.2014.08.003.

URL http://linkinghub.elsevier.com/retrieve/pii/ S0191261514001386

925

[79] M. Twarogowska, P. Goatin, R. Duvigneau, Comparative Study of Macroscopic Pedestrian Models, Transportation Research Procedia 2 (2014) 
477-485. doi:10.1016/j.trpro.2014.09.063.

URL http://dx.doi.org/10.1016/j.trpro.2014.09.063

[80] G. G. Løvås, Modeling and simulation of pedestrian traffic flow, Transportation Research Part B: Methodological 28 (6) (1994) 429-443. doi : $10.1016 / 0191-2615(94) 90013-2$.

[81] K. Drager, G. Løvås, J. Wiklund, Evacsim: A comprehensive evacuation simulation tool, in: J. Sullivan (Ed.), Proceedings of the 1992 International Emergency Management and Engineering Conference, 1992, pp. $101-108$.

[82] G. G. Løvås, Models of wayfinding in emergency evacuations, European Journal of Operational Research 105 (3) (1998) 371-389. doi:10.1016/ S0377-2217 (97) 00084-2.

[83] R. Y. Guo, H. J. Huang, S. C. Wong, Collection, spillback, and dissipation in pedestrian evacuation: A network-based method, Transportation Research Part B: Methodological 45 (3) (2011) 490-506. doi: $10.1016 / j . t r b .2010 .09 .009$.

URL http://dx.doi.org/10.1016/j.trb.2010.09.009

[84] V. J. Blue, J. L. Adler, Cellular automata microsimulation for modeling bi-directional pedestrian walkways, Transportation Research Part B: Methodological 35 (3) (2001) 293-312. doi:10.1016/S0191-2615(99) 00052-1.

[85] R. Y. Guo, H. J. Huang, S. C. Wong, Route choice in pedestrian evacuation under conditions of good and zero visibility: Experimental and simulation results, Transportation Research Part B: Methodological 46 (6) (2012) 669-686. doi:10.1016/j.trb.2012.01.002.

URL http://dx.doi.org/10.1016/j.trb.2012.01.002

[86] L. a. Pereira, L. H. Duczmal, F. R. B. Cruz, Congested emergency evacuation of a population using a finite automata approach, Safety Science 
51 (1) (2013) 267-272. doi:10.1016/j.ssci.2012.07.002.

URL http://dx.doi.org/10.1016/j.ssci.2012.07.002

[87] G. Antonini, M. Bierlaire, M. Weber, Discrete choice models of pedestrian walking behavior, Transportation Research Part B: Methodological 40 (8) (2006) 667-687. doi:10.1016/j.trb.2005.09.006.

960

[94] P. a. Thompson, E. W. Marchant, Testing and application of the computer model SIMULEX, Fire Safety Journal 24 (2) (1995) 149-166. 
doi:10.1016/0379-7112(95)00020-T.

URL http://linkinghub.elsevier.com/retrieve/pii/ control and differential games, Optimal Control Applications and Methods 24 (3) (2003) 153-172. doi:10.1002/oca. 727. 
1010

1015

1020

[104] H. Nelson, H. MacLennan, Emergency Movement, in: P. DiNinno, et al. (Eds.), SFPE Handbook of Fire Protection Engineering, 2nd Edition, National Fire Protection Association, Quincy, Mass, 1995.

[105] J. Brown, The Knapsack Sharing Problem, Operations Research 27 (2) 1025

[101] S. P. Hoogendoorn, P. H. L. Bovy, Pedestrian route-choice and activity scheduling theory and models, Transportation Research Part B: Methodological 38 (2) (2004) 169-190. doi:10.1016/S0191-2615(03)00007-9.

[102] C. O. Tong, S. C. Wong, A predictive dynamic traffic assignment model in congested capacity-constrained road networks, Transportation Research Part B: Methodological 34 (8) (2000) 625-644. doi:10.1016/ S0191-2615(99) 00045-4.

[103] A. Lachapelle, M. T. Wolfram, On a mean field game approach modeling congestion and aversion in pedestrian crowds, Transportation Research Part B: Methodological 45 (10) (2011) 1572-1589. doi:10.1016/j.trb. 2011.07 .011$.

(1979) 341-355. 